\title{
The straw man of prescribing in psychiatry ${ }^{\dagger}$
}

COMMENTARY ON... DRUG-CENTRED PSYCHOPHARMACOLOGY

\section{David Taylor}

\begin{abstract}
SUMMARY
'Drug-centred' prescribing in psychiatry has been proposed as a new and better approach than the current 'disease-centred' alternative. It targets symptoms most important to the patient using the concept that psychotropics act indirectly by altering normal functioning. I contend that it is a straw man: psychiatrists already use 'drugcentred' prescribing, applying their knowledge of a drug's pharmacological profile and evidence base to treat each patient's symptoms, not their diagnostic classification. Furthermore, there is no compelling evidence that psychotropics act by altering 'normal' functioning: rather, they have diverse effects on different mental states.
\end{abstract}

\section{DECLARATION OF INTEREST}

Advisory Board member for: Lundbeck, Servier, Sunovion; lectures for Janssen, Lundbeck, Otsuka, Servier: research funding from: BMS, Janssen, Lundbeck

Yeomans and colleagues (2015, this issue) tell us that today's prescribers first diagnose and then, on the basis of their knowledge of the underlying biochemical pathology of the specific disorder identified, choose a drug which directly or indirectly reverses that biochemical abnormality, thus helping to 'restore the body to a more normal or healthy state' (I hope I have not overstated this). Thus their contention is that (a) diagnosis is critical for drug selection, (b) the underlying biochemical abnormality associated with any diagnosis is reasonably well characterised and understood, and (c) prescribers select drugs on the basis that their pharmacological actions are in opposition to the known biochemical abnormality of the condition diagnosed (the model sold to them by the drug manufacturers). This argument is, of course, a 'straw man' of rather large proportions.

One of the easiest and best ways to win an argument is, figuratively, to erect a straw man and then to knock it down. Ideally, the straw man must have the look of your opposition's point of view, but must be subtly amended to render it superficially, if only faintly, preposterous and, on closer examination, utterly untenable. The straw man argument often reveals much about the relative merits of the two sides of the debate. The erectors of the straw man must at some level know that their opposer's position has some merit and might be rational and cogent: why else change it into something ludicrous?

\section{The straw man}

I suspect that no prescriber examines a patient, diagnoses them with, say, schizophrenia, infers that they have a surfeit of dopamine and then decides to block the action of dopamine with an antipsychotic, expecting full resolution of symptoms. It is more likely that the prescriber notes various psychotic symptoms and prescribes a drug that she hopes might improve to some extent some of the symptoms to the patient's benefit. A benzodiazepine might relieve distress, an antipsychotic might lessen the frequency of auditory hallucinations, promethazine, a straightforward sedative, might reduce physical hyperactivity, for example.

Psychiatrists, I contend, prescribe drugs to treat symptoms, not diseases or artificially defined conditions. Rarely is there any certainty about diagnosis and more rarely still do prescribers have the faintest clue of the indications for which individual drugs are formally licensed. Who knows that paliperidone palmitate is not indicated for schizoaffective disorder, that valproate semisodium is licensed in Europe only for acute treatment of mania, that sodium valproate and valproic acid are not licensed for any psychiatric disorder? This off-licence prescribing is clear evidence of a 'drug-centred' approach, as Yeomans et al point out, but prescribing within a drug's licence is not, in my view, evidence of the use of a disease-centred model, the matching of condition and indication more often being mere coincidence.
David Taylor is Director of Pharmacy and Pathology at the Maudsley Hospital and Professor of Psychopharmacology at King's College London. Correspondence Professor David Taylor, Pharmacy Department, Maudsley Hospital, Denmark Hill, London SE5 8AZ, UK. Email: david. taylor@slam.nhs.uk

${ }^{\dagger}$ See pp. 229-236, this issue. 
There are a few areas of psychiatry in which a clear diagnosis is crucial to prescribing decisions. Unipolar and bipolar depression have similar symptoms (Akiskal 2000) but respond very differently to pharmacotherapy - an antidepressant is the treatment of choice for the former but is perhaps best avoided in the latter (Taylor 2015). Importantly, however, different prescribing practices for these two disorders are dictated by observed clinical outcomes of trials rather than by any particular theory of 'biochemical imbalance'. Nobody really knows why, say, aripiprazole has therapeutic activity in unipolar depression (Berman 2009) but not in bipolar depression (Taylor 2014).

\section{Drug safety}

In my experience, prescribers do not have 'an uncritical assumption that most medication is restorative and safe'. Far from it. Who can fail to recognise the myriad dangers of, say, haloperidol or fluoxetine or clozapine? And, while we're at it, what might clozapine be restoring? Nobody knows, but clozapine still gets prescribed, often to considerable effect, to patients with all kinds of 'diseases' (Kane 1988; Suppes 1999; Frogley 2013). There is no disease-centred approach evident here, and given that we do not know how clozapine exerts its therapeutic effects, it cannot be prescribed on the basis that it is restoring the balance of some chemical or other.

Yeomans et al also tell us that 'patients' complaints about [the psychoactive effects of antipsychotics] are still frequently minimised or dismissed'. I am sure this does occur, although one hopes to a lesser extent than in earlier times. Why this attitude should be blamed on a diseasecentred model is unclear and not substantiated. This observation is also used to suggest that, in some cases, the benefits of antipsychotics (reduced relapse) might be outweighed by their negative effects. Of course, this is true, but we need to be careful about the proportion of people for whom this is the case. The Wunderink paper (Wunderink 2013) cited is an outlier among a large number of studies (e.g. Hough 2010; Kane 2012; Emsley 2014) that indicate that the exact opposite of its findings are more commonly seen. Almost every discontinuation study ever undertaken shows relapse rates of $60-80 \%$ at 1 year and $>90 \%$ at 2 years. Surely the best way to balance the positive and negative effects of psychotropics is to find a drug that works while giving rise to the least frequent or least severe adverse effects (or whatever one might want to call them).

\section{Collaborative prescribing}

No one would argue that collaboration is not a good thing when prescribing psychotropics to patients. For this to happen we do not, I contend, need to believe that 'drugs act through altering normal mental functions', whatever normal mental functions may be. We need only to have in mind the patient's best interests. Nor is it always a good idea to prescribe according to 'patient-identified goals' (although it often is) - the nature of many mental illnesses is such that these goals might well be against the patient's interest were they not psychotic, manic or depressed. To take a trite example, a patient's goal might be to be properly recognised as the saviour of mankind. Should one prescribe according to this goal? There are times when such decisions are best made by someone other than the patient.

\section{Reinventing the wheel?}

That a drug-centred approach is already employed by psychiatrists is perhaps best exemplified by the use of quetiapine. This is a drug with multiple pharmacological actions and metabolised to at least four similar compounds with markedly different pharmacological actions (Fisher 2012). It is licensed in Europe for schizophrenia, bipolar mania, bipolar depression and unipolar depression, and is also active in generalised anxiety disorder (Mezhebovsky 2013), for which it is not licensed. Quetiapine is prescribed for symptoms related to those conditions on the basis that it has been shown (in trials and in practice) to be effective. I contend that it is not prescribed on the basis of its pharmacological actions, because no one could possibly conceive of the multitude of pharmacological permutations which arise from the ingestion of a single dose of quetiapine, even less reckon them against the imagined biochemical changes claimed to be associated with the condition diagnosed.

So, in essence, we agree: drugs should be prescribed to treat symptoms on the basis of prior observations that such symptoms are improved by particular drugs. There is a need to be broadly aware of a drug's pharmacological profile (but not its socalled mode of action - which is often not known anyway) for the purposes of predicting what specific effects might arise (e.g. blocking dopamine seems to improve psychosis but also raises prolactin). In fact, this approach has recently been formalised by the 'neuroscience-based nomenclature' (NbN) group (Zohar 2014), who seek to classify drugs according to their actions rather than their usage. So, for example, quetiapine is referred to not as an 
antipsychotic but as a dopamine multifunctional receptor antagonist (DAmF-RAn), better reflecting its pharmacological actions. Importantly, $\mathrm{NbN}$ does not suggest that these actions used to identify or classify a drug are in any way reversing known abnormalities associated with any condition. $\mathrm{NbN}$ simply recognises that psychotropics have a variety of pharmacological actions that can render them useful in a range of different conditions - or, to put it another way, that prescribing should be undertaken on the basis of what the drug does rather than what the condition is. A drug-based model, if you like.

\section{There must be a mode of action}

An important aspect to the proposed drug-centred approach is the observation that drugs exert effects on normal mental functioning. An extension to this theory, it seems, is that it is this alteration to 'normal' functioning that brings about a drug's apparent therapeutic effects. The mounting implausibility inherent in adding one supposition to another has the effect of rendering the whole proposal difficult to accept. We can agree that concentration on the drug rather than the diagnosis is probably to the patient's benefit. However, can we agree that all psychotropics cause alterations in normal mental functioning? Possibly, although it is worth remembering that many psychotropics do very different things in 'normal' people than in mentally distressed people - selective serotonin reuptake inhibitors (SSRIs) improve mood in the depressed but not in the euthymic; haloperidol cannot abolish auditory hallucinations unless they are experienced in the first place. This observation that psychotropics cause different effects in those considered mentally ill is not consistent with the suggestion that drugs act by changing 'normal' mental functioning. Surely if this were the case, the effects would be the same in everyone. Likewise, the idea that these changes to functioning are (entirely?) responsible (again, I hope I am not overstating the theory here) for a drug's therapeutic effects seems implausible. Sure, clozapine (for example) will sedate and render a person impassive to what is going on around them, but these effects alone are not nearly enough to account for the changes frequently seen in delusional content, paranoia, thought disorder, hallucinations and everyday functioning. Clozapine, as with other drugs, must be doing something specific and direct; it must have a mode of action, if only we knew what it was.

\section{Conclusions}

I see no difficulty accepting the drug-centred approach because that is what is already being done. Likewise, targeting symptoms or problems most important to the patient seems only right and proper, notwithstanding the likelihood that, in some cases, the patient's wishes will not be in their best interests. But I cannot accept that psychotropics act indirectly by altering normal functioning: aside from the fact that normal functioning is difficult to define, there is much too much evidence demonstrating that psychotropics have diverse effects in people with different, what might be loosely termed, mental conditions.

\section{References}

Akiskal HS, Bourgeois ML, Angst J, et al (2000) Re-evaluating the prevalence of and diagnostic composition within the broad clinical spectrum of bipolar disorders. Journal of Affective Disorders, 59 (suppl 1): S5-30.

Berman RM, Fava M, Thase ME, et al (2009) Aripiprazole augmentation in major depressive disorder: a double-blind, placebo-controlled study in patients with inadequate response to antidepressants. CNS Spectrums, 14: 197-206

Emsley R, Chiliza B, Asmal L, et al (2014) A randomized, controlled trial of omega-3 fatty acids plus an antioxidant for relapse prevention after antipsychotic discontinuation in first-episode schizophrenia. Schizophrenia Research, 158: 230-35.

Fisher DS, Handley SA, Flanagan RJ, et al (2012) Plasma concentrations of quetiapine, N-desalkylquetiapine, o-desalkylquetiapine, 7-hydroxyquetiapine, and quetiapine sulfoxide in relation to quetiapine dose, formulation, and other factors. Therapeutic Drug Monitoring, 34: 415-21.

Frogley C, Anagnostakis K, Mitchell S, et al (2013) A case series of clozapine for borderline personality disorder. Annals of Clinical Psychiatry, 25: $125-34$.

Hough D, Gopal S, Vijapurkar U, et al (2010) Paliperidone palmitate maintenance treatment in delaying the time-to-relapse in patients with schizophrenia: a randomized, double-blind, placebo-controlled study. Schizophrenia Research, 116: 107-17.

Kane J, Honigfeld G, Singer J, et al (1988) Clozapine for the treatmentresistant schizophrenic: a double-blind comparison with chlorpromazine. Archives of General Psychiatry, 45: 789-96.

Kane JM, Sanchez R, Perry PP, et al (2012) Aripiprazole intramuscular depot as maintenance treatment in patients with schizophrenia: a 52week, multicenter, randomized, double-blind, placebo-controlled study. Journal of Clinical Psychiatry, 73: 617-24.

Mezhebovsky I, Magi K, She F, et al (2013) Double-blind, randomized study of extended release quetiapine fumarate (quetiapine XR) monotherapy in older patients with generalized anxiety disorder. International Journal of Geriatric Psychiatry, 28: 615-25.

Suppes T, Webb A, Paul B, et al (1999) Clinical outcome in a randomized 1 -year trial of clozapine versus treatment as usual for patients with treatment-resistant illness and a history of mania. American Journal of Psychiatry, 156: 1164-69.

Taylor DM, Cornelius V, Smith L, et al (2014) Comparative efficacy and acceptability of drug treatments for bipolar depression: a multipletreatments meta-analysis. Acta Psychiatrica Scandinavica, 130: 452-69.

Taylor D, Paton C, Kapur S (2015) Maudsley Prescribing Guidelines in Psychiatry (12th edn). Wiley-Blackwell.

Wunderink L, Nieboer RM, Wiersma D, et al (2013) Recovery in remitted first-episode psychosis at 7 years of follow-up of an early dose reduction/ discontinuation or maintenance treatment strategy: long-term follow-up of a 2-year randomized clinical trial. JAMA Psychiatry, 70: 913-20.

Yeomans D, Moncrieff J, Huws R (2015) Drug-centred psychopharmacology: a non-diagnostic framework for drug treatment. BJPsych Advances, 21: 229-36.

Zohar J, Stahl S, Moller H-J, et al (2014) Neuroscience-Based Nomenclature. Cambridge University Press. 